ACCEPTED BY APJ

Preprint typeset using IATEX style emulateapj v. 11/12/01

\title{
PERIODIC THERMONUCLEAR X-RAY BURSTS FROM GS 1826-24 AND THE FUEL COMPOSITION AS A FUNCTION OF ACCRETION RATE
}

\author{
Duncan K. Galloway ${ }^{1}$, Andrew Cumming ${ }^{2,3}$, Erik Kuulkers ${ }^{4}$, Lars Bildsten ${ }^{5}$, Deepto \\ Chakrabarty $^{1,6,7}$, AND Richard E. Rothschild ${ }^{8}$ \\ Accepted by ApJ
}

\begin{abstract}
We analyze 24 type I X-ray bursts from GS 1826-24 observed by the Rossi X-ray Timing Explorer between 1997 November and 2002 July. The bursts observed between 1997-98 were consistent with a stable recurrence time of $5.74 \pm 0.13 \mathrm{hr}$. The persistent intensity of GS 1826-24 increased by $36 \%$ between 1997-2000, by which time the burst interval had decreased to $4.10 \pm 0.08 \mathrm{hr}$. In 2002 July the recurrence time was shorter again, at $3.56 \pm 0.03 \mathrm{hr}$. The bursts within each epoch had remarkably identical lightcurves over the full $\approx 150 \mathrm{~s}$ burst duration; both the initial decay timescale from the peak, and the burst fluence, increased slightly with the rise in persistent flux. The decrease in the burst recurrence time was proportional to $\dot{M}^{-1.05 \pm 0.02}$ (assuming that $\dot{M}$ is linearly proportional to the X-ray flux), so that the ratio $\alpha$ between the integrated persistent and burst fluxes was inversely correlated with $\dot{M}$. The average value of $\alpha$ was $41.7 \pm 1.6$. Both the $\alpha$ value, and the long burst durations indicate that the hydrogen is burning during the burst via the rapid-proton (rp) process. The variation in $\alpha$ with $\dot{M}$ implies that hydrogen is burning stably between bursts, requiring solar metallicity $(Z \sim 0.02)$ in the accreted layer. We show that solar metallicity ignition models naturally reproduce the observed burst energies, but do not match the observed variations in recurrence time and burst fluence. Low metallicity models $(Z \sim 0.001)$ reproduce the observed trends in recurrence time and fluence, but are ruled out by the variation in $\alpha$. We discuss possible explanations, including extra heating between bursts, or that the fraction of the neutron star covered by the accreted fuel increases with $\dot{M}$.
\end{abstract}

Subject headings: stars: individual (Ginga 1826-238, GS 1826-24) — X-rays: bursts

\section{INTRODUCTION}

Studies of recurrence times and energetics of type I Xray bursts in low-mass X-ray binaries (LMXBs) led to one of the most compelling arguments for their origin as unstable thermonuclear burning of accreted hydrogen and helium (e.g. Strohmayer \& Bildsten 2003). Long duration broadband X-ray observations with satellites in high orbits such as EXOSAT allowed high-precision measurements of source fluxes and uninterrupted trains of X-ray bursts (e.g. Gottwald et al. 1986; Langmeier et al. 1987). In these sequences of bursts, the ratio of persistent fluence between bursts $E_{\mathrm{p}}$ to burst fluence $E_{\mathrm{b}}$,

$$
\alpha \equiv \frac{E_{\mathrm{p}}}{E_{\mathrm{b}}}=\frac{\int_{0}^{\Delta t} F_{\mathrm{p}} d t}{\int_{0}^{\Delta t} F_{\mathrm{b}} d t},
$$

where $F_{\mathrm{p}}$ is the persistent flux from accretion, $F_{\mathrm{b}}$ is the burst flux, and $\Delta t$ is the interval from the beginning of one burst to the next, was found to be $\sim 10-100$. This is consistent with the energy release in bursts coming from nuclear burning, which gives $\approx 1.6 \mathrm{MeV}(5 \mathrm{MeV})$ per nucleon when burning pure helium (solar abundance material) to heavy elements, compared to $\approx 200 \mathrm{MeV}$ per nucleon from gravitational energy release due to accretion. The general agreement of measured $\alpha$ values with this picture was an important confirmation of the thermonuclear burst model.

Detailed comparisons of observations and theory have had mixed success, however, particularly attempts to reconcile the observed trends in burst behavior with accretion rate with those expected from theory (Fujimoto et al. 1987; van Paradijs et al. 1988; Bildsten 2000; Cornelisse et al. 2003; Cumming 2003). Unfortunately, observational studies are now more difficult because most of the active broadband X-ray observatories are in low Earth orbits. Interruptions in the observations due to occultations and passages through localised high-background regions occur at the satellite orbital period, which is of the same order as the typical recurrence time for bursts.

Despite such obstacles, GS 1826-24 (also known as Ginga $1826-238 ; l=9.27, b=-6.09$ ) is an ideal subject for studying X-ray bursts due to its bright, regular and frequent bursts. The source was discovered as a new transient by Ginga (Tanaka 1989). Conclusive evidence of the presence of a neutron star was obtained with the BeppoSAX detection of thermonuclear bursts (Ubertini et al.

1 Center for Space Research, Massachusetts Institute of Technology, Cambridge, MA 02139, email: duncan@space.mit.edu

2 Dept. of Astronomy and Astrophysics, University of California, Santa Cruz, CA 95064; email: cumming@ucolick.org

3 Hubble Fellow

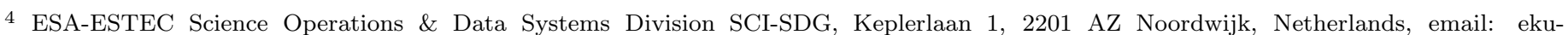
ulker@rssd.esa.int

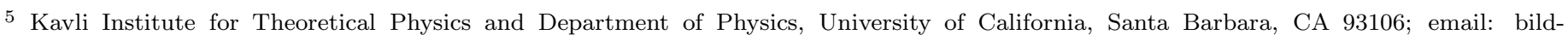
sten@kitp.ucsb.edu

6 also Department of Physics, Massachusetts Institute of Technology, email: deepto@space.mit.edu

7 Alfred P. Sloan Research Fellow

8 Center for Astrophysics and Space Science, University of California, San Diego, La Jolla, CA 92093, email: rrothschild@ucsd.edu 
1997), although this source may also have been the origin of X-ray bursts observed much earlier by OSO-8 (Becker et al. 1976). Optical photometry of the $V=19$ counterpart revealed a $2.1 \mathrm{hr}$ modulation, as well as optical bursts (Homer et al. 1998). The delay time measured between the X-ray and optical bursts is consistent with the binary separation for a $2.1 \mathrm{hr}$ orbit (see also Kong et al. 2000). Based on optical measurements, the distance, $d$, to the source is at least $4 \mathrm{kpc}$ (Barret et al. 1995). An upper limit of $8 \mathrm{kpc}$ has been derived from the peak fluxes of bursts measured by BeppoSAX, ASCA and RXTE (in 't Zand et al. 1999; Kong et al. 2000), which would position the source just outside the Galactic bulge. The global accretion rate inferred from the observed flux is then $\dot{M} \approx 10^{-9} M_{\odot} \mathrm{yr}^{-1}\left(F_{\mathrm{p}} / 3 \times 10^{-9} \mathrm{ergs} \mathrm{cm}^{-2} \mathrm{~s}^{-1}\right)(d / 6 \mathrm{kpc})^{2}$, about a factor of ten higher than the expected timeaveraged accretion rate for a NS orbiting a main sequence star in a $2.1 \mathrm{hr}$ orbit (Verbunt \& van den Heuvel 1995). Such a discrepancy is not surprising for a transient accretor.

Extensive BeppoSAX Wide Field Camera (WFC) observations between 1996 August and 1998 October revealed around 70 bursts, which recurred on an average interval of $5.76 \pm 0.62 \mathrm{hr}$ (leading to the pseudonym "the Clocked burster"; Ubertini et al. 1999). Analysis of the entire WFC sample of 260 bursts revealed that the source consistently exhibits approximately periodic bursts with a recurrence time which decreases significantly as the persistent flux increases (Cornelisse et al. 2003). Such regularity of bursts over a long time span is not observed in other sources and implies a remarkably stable accretion rate, as well as complete burning of the accreted fuel and a constant fuel covering fraction.

Based on the decay time scale of the bursts observed by RXTE (Kong et al. 2000) and the recurrence times, Bildsten (2000) suggested that the bursts must involve mixed hydrogen/helium $(\mathrm{H} / \mathrm{He})$ burning. In this scenario, the initial helium flash generates seed nuclei for hydrogen burning, which gives rise to an additional energy release and also allows an active rapid proton (rp) process of Wallace \& Woosley (1981). This process burns hydrogen by successive proton capture reactions on seed nuclei that subsequently $\beta$-decay. These reactions move the seed nuclei up the proton-rich side of the periodic table (much like the r-process which occurs by neutron captures on the neutron-rich side of the periodic table). Theoretical work indicates that the end point of the rp-process is set either by the complete consumption of the available hydrogen (Hanawa et al. 1983; Rembges et al. 1997; Schatz et al. 1998; Koike et al. 1999) or by reaching the closed SnSbTe cycle found by Schatz et al. (2001). Uncertainties still remain in the actual total time to burn the matter, since the $\beta$-decay lifetimes of many of these proton-rich nuclei are not known experimentally. Thus, GS $1826-24$ is an ideal candidate to test, and provide input for, our current theoretical understanding of the role of the rp-process in thermonuclear X-ray bursts.

We present new measurements of the burst recurrence time in GS 1826-24 with the Rossi X-ray Timing Explorer (RXTE). We first compare the properties of different bursts to highlight their unusually uniform properties. We examine the long-term trend of burst recurrence time as a function of persistent flux, and test for variations in the $\alpha$-value within different epochs. By comparing to theoretical models of type I bursts, we are able to constrain the metallicity of the accreting material, and the fraction of the neutron star's surface over which accretion takes place.

\section{OBSERVATIONS}

We analysed observations of GS $1826-24$ by the All Sky Monitor (ASM; Levine et al. 1996), the Proportional Counter Array (PCA; Jahoda et al. 1996) and the Highenergy X-ray Timing Experiment (HEXTE; Rothschild et al. 1998) aboard RXTE. The ASM consists of three Scanning Shadow Cameras (SSCs) sensitive to 2-10 keV photons mounted on a rotating platform, which makes $90 \mathrm{~s}$ observations ("dwells") covering most of the sky every few hours. Data from each SSC from each dwell are averaged to obtain the daily intensities of all known sources in the field of view. The PCA is made up of 5 identical co-aligned Proportional Counter Units (PCUs) sensitive to photons in the energy range $2-60 \mathrm{keV}$, and with a total area of $\approx 6000 \mathrm{~cm}^{2}$. Data is collected during pointed observations in standard data modes (1 and 2), as well as a variety of user defined modes offering time resolution up to $1 \mu \mathrm{s}$ and 256 energy channels. The HEXTE comprises two clusters with $1600 \mathrm{~cm}^{2}$ total area, each of which contains 4 scintillation detectors collimated to view a common direction and sensitive to photons in the range $15-250 \mathrm{keV}$.

We searched for X-ray bursts in 1-s binned "Standard1 " mode data from 43 public $R X T E / \mathrm{PCA}$ observations of GS 1826-24 between 1997 November 5 and 2000 September 27 , totalling $347 \mathrm{ks}$. We also searched in a $30 \mathrm{ks}$ proprietary observation from 2002 July 27 (observation ID 70044, PI: Rothschild). We identified 24 bursts in total. In three cases the PCUs were switched on during what appeared to be the decay from a burst. We estimated the start time of these burst candidates by comparing the decay light curve with the profile from the burst immediately before or after. While no blackbody temperature decrease was observed in any of these three cases, no decrease was observed for the other bursts when at the same stage. Since the observed profiles of the other bursts are so uniform, we expect the inferred start times to be correct to within 5-10 s. In a fourth case the burst began while the satellite was still slewing to the source. For that burst the measurement of the peak flux and fluence are suspect, but the start time was precisely measured. For the analysis presented here, we include these four bursts for calculations of recurrence times but exclude them from any analysis involving measurements of the flux or fluence.

For each burst where we observed the entire profile we extracted PCA spectra binned initially at $0.25 \mathrm{~s}$ (with progressively larger bins towards the tail of the burst) from Event mode data (125 $\mu$ s time resolution and 64 energy channels). We calculated a separate response matrix for each burst using PCARSP version 8.0 (supplied with LHEASOFT version 5.2, 2002 June 25). We fitted an absorbed blackbody model to each spectrum, using a persistent spectrum extracted from a 16-s interval before the burst as background. This approach is relatively 


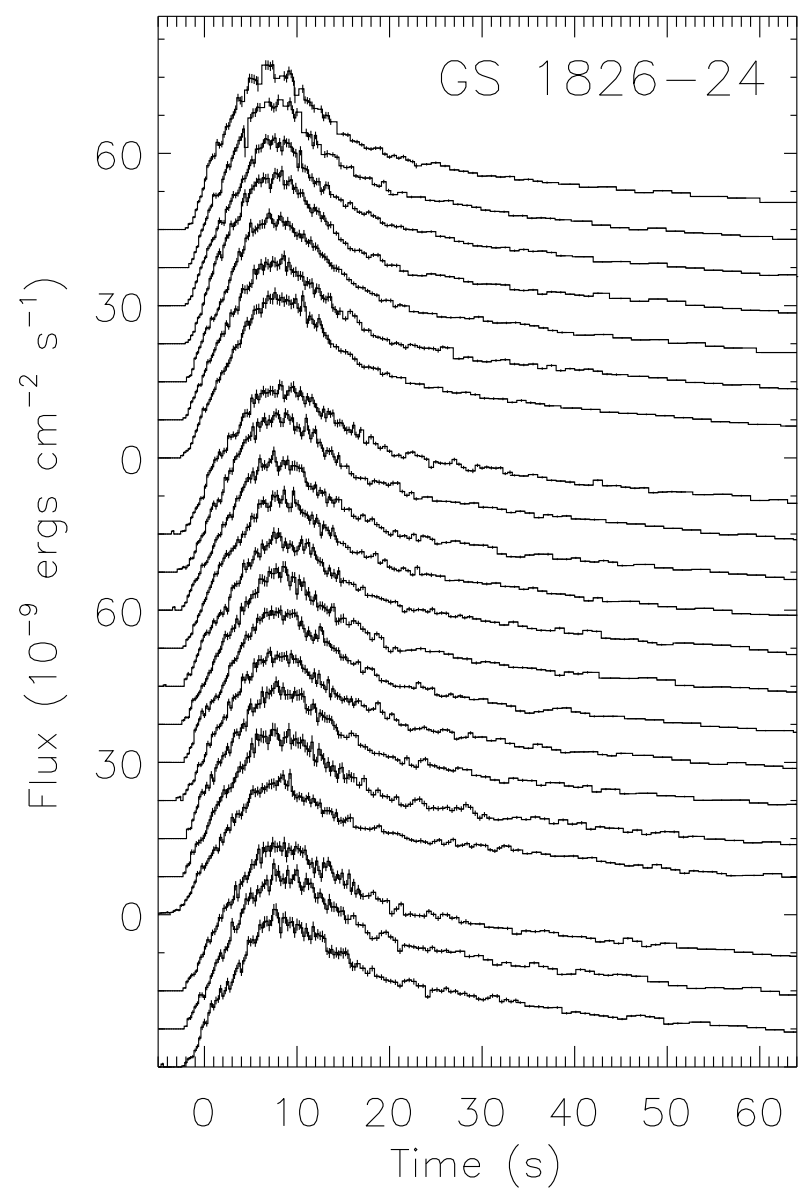

Fig. 1.- Profiles of 20 X-ray bursts from GS 1826-24 observed by $R X T E$ between $1997-2002$, plotted with varying vertical offsets for clarity. The upper group of 7 bursts were observed in 1997-98, the middle group of 10 bursts in 2000, while the lower group of 3 were observed in 2002. The bursts from each epoch have been timealigned by cross-correlating the first 8 seconds of the burst. Error bars indicate the $1 \sigma$ uncertainties.

standard for X-ray burst analysis (e.g. Kuulkers et al. 2002). We estimated the bolometric burst flux at each timestep as $F_{\mathrm{bol}, i}=1.0763 \times$ $10^{-11} T_{\mathrm{bb}, i}^{4} K_{\mathrm{bb}, i} \quad \operatorname{ergs~cm}^{-2} \mathrm{~s}^{-1}$, from the blackbody (color) temperature $T_{\mathrm{bb}, i}$ and normalization $K_{\mathrm{bb}, i}=$ $\left(R_{\mathrm{bb}} / d_{10 \mathrm{kpc}}\right)^{2}$, where $R_{\mathrm{bb}}$ is the apparent photosphere radius in $\mathrm{km}$, and $d_{10 \mathrm{kpc}}$ the distance to the source in units of $10 \mathrm{kpc}$. We define the burst start as the time at which the bolometric flux exceeds $25 \%$ of the peak flux, and the rise time as the interval for the flux to subsequently exceed $90 \%$ of the maximum. We fitted the flux decay to an exponential profile with a "break" at which point the $e$-folding time is allowed to vary discontinuously. We integrated the measured fluxes over $\approx 150 \mathrm{~s}$ covering the burst, and extrapolated beyond this based on the exponential fits, to derive the fluence.

We also estimated the instrumental background using PCABACKEST version 3.0 and the "combined" bright source models, and measured the (absorbed) persistent 2.5-25 keV PCA flux by integrating over an absorbed blackbody plus power law model fitted to the persistent (pre-burst) spectra. The mean reduced- $\chi^{2}$ for the persistent spectral fits was 1.07 (56 degrees of freedom). The neutral column density was in most cases poorly con- strained and not significantly different from zero, and in the mean was $n_{\mathrm{H}}=(2.4 \pm 1.4) \times 10^{22} \mathrm{~cm}^{-2}$. While this model provided a good fit to the PCA data alone, combined fits including the HEXTE spectrum additionally required modelling of the high-energy spectral cutoff (see section $§ 3.2)$.

\section{BURST PROFILES, ENERGETICS AND RECURRENCE TIMES}

The X-ray bursts observed by $R X T E$ were remarkably similar to each other (Fig. 1). The rise times were relatively long, betseen 4.75 and $7 \mathrm{~s}(5.6 \pm 0.6 \mathrm{~s}$ on average). The first exponential decay timescale increased from $14.7 \pm 0.7$ to $17.5 \pm 1.1 \mathrm{~s}$ between the $1997-98$ and 2000 bursts, and to $19.1 \pm 1.3$ for the 2002 bursts. The variation of the burst profile with epoch is obvious in the averaged lightcurves (Fig. 2). The second exponential timescale was, on average, $43 \pm 1$ s. The peak fluxes also showed weak evidence for a decrease with time; the mean for the 7 bursts observed in 1997-98 was $(33.0 \pm 0.8) \times 10^{-9} \mathrm{ergs} \mathrm{cm}^{-2} \mathrm{~s}^{-1}$, while for the bursts observed in 2000-2 it was $(30.5 \pm 1.1) \times 10^{-9} \mathrm{ergs} \mathrm{cm}^{-2} \mathrm{~s}^{-1}$ (note that the averages of burst properties calculated here exclude the bursts which we did not observe in their entirety). This decrease was substantially larger than the variation in the pre-burst persistent emission (see $\S 3.1$, below). Thus, it appears unlikely that the observed variation in the peak burst flux arose as a side-effect of subtracting the persistent emission as background. The net effect of the variations in peak flux and timescale was to keep the fluence approximately constant, at $\approx 1.1 \times 10^{-6} \mathrm{ergs} \mathrm{cm}^{-2}$. None of the bursts exhibited evidence for radius expansion, so that the maximum burst flux is a lower limit to the Eddington luminosity. The implied distance limit is consistent with that derived from previous observations.

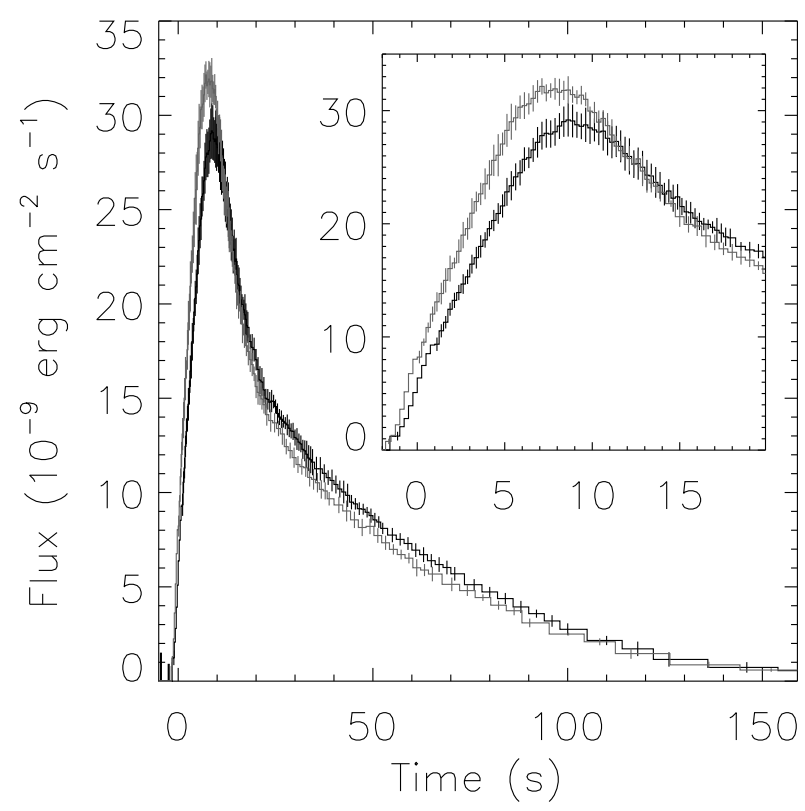

FIG. 2.- Mean profiles of 7 X-ray bursts from GS 1826-24 observed by RXTE during 1997-98 (grey histogram), and of 10 bursts observed during 2000 (black histogram). The bursts from 2002 have similar profiles to those from 2000. Error bars indicate the $1 \sigma$ uncertainties, derived from the scatter of the flux within each time bin over all the bursts. The inset shows the same profiles, expanded to show more detail around the burst rise and peak. 

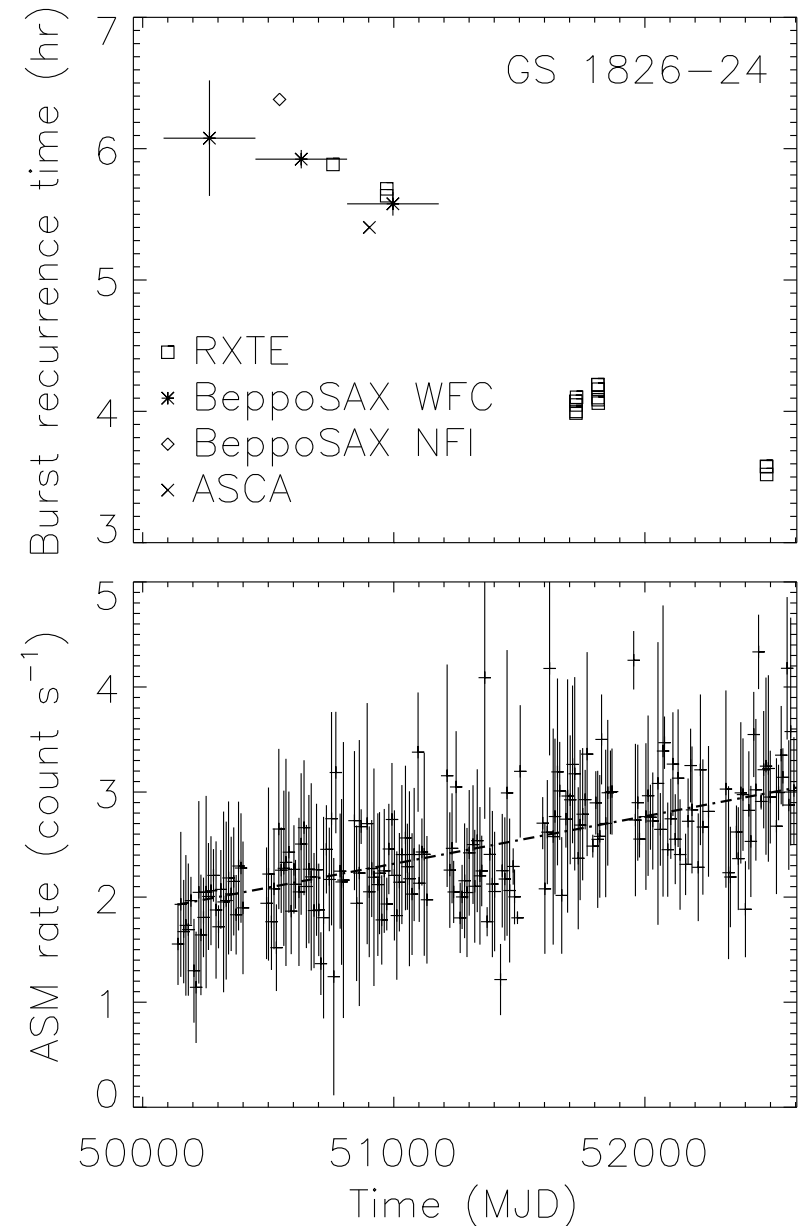

FIG. 3.- (top panel) Recurrence time for thermonuclear bursts from GS 1826-24. Shown are the 1997-2002 measurements by $R X T E$ (open squares), as well as earlier measurements by BeppoSAX (in 't Zand et al. 1999; Cocchi et al. 2001) and $A S C A$ (Kong et al. 2000). (bottom panel) Long-term ASM count rate history of GS 1826-24. 10-day averages were calculated from 1-day measurements, excluding those points with errors $>0.7$ count $\mathrm{s}^{-1}$. The dot-dashed line shows the linear fit curve. The linear trend is significant at the $10 \sigma$ level.

\subsection{Long-term burst interval history}

Due to its low Earth orbit, RXTE can typically only observe any given source for $65 \%$ of each 90 min orbit. Thus, it is likely that some bursts were missed during the gaps between observations. The shortest burst intervals were found between pairs of bursts observed on 1997 November 5-6, 2000 Jun 30, and 2002 July 29 , at $\Delta t=5.88$, $4.00 \mathrm{hr}$, and $3.58 \mathrm{hr}$, respectively. While the first value is consistent with other measurements around the same time (e.g. Ubertini et al. 1999; Cocchi et al. 2001), the latter two are almost a third shorter. The other burst intervals measured from the $R X T E$ observations are at least a factor of 2 greater than the shortest intervals, and were close to integer multiples of the two shortest intervals in each epoch. Furthermore, in each of the longer burst intervals, the predicted intermediate burst times (assuming regular burst occurrence) fell within data gaps. Thus, it was still possible for the bursts to be recurring on a regular timescale.
In order to independently test for the presence of these intermediate bursts, we examined ASM dwells during these longer intervals. Very few dwells occurred close to the predicted time of the bursts. Thus, we found no evidence for bursts at the predicted intermediate times; on the other hand, with the present data we cannot exclude the presence of bursts within these gaps. Taking into account these missed bursts, we conclude that the observed burst times are consistent with regular recurrence intervals of $5.74 \pm 0.13 \mathrm{hr}$ in $1997-98,4.10 \pm 0.08 \mathrm{hr}$ in 2000 , and $3.56 \pm 0.03 \mathrm{hr}$ in 2002 .

When combined with BeppoSAX and ASCA observations between 1996-98, as well as more recent observations with $R X T E$, these measurements provide evidence for a long-term, steady decrease in $\Delta t$ with time (Fig. 3 ). Over the same interval, the 10-d averaged 2-10 keV ASM intensity measurements of GS 1826-24 show evidence for a long-term trend of increasing persistent flux. This trend was confirmed by the PCA measurements. The 2.5-25 keV persistent flux measured during 1997-98 was $(1.32 \pm 0.05) \times 10^{-9} \mathrm{ergs} \mathrm{cm}^{-2} \mathrm{~s}^{-1}$, while in 2000 it was $(1.80 \pm 0.05) \times 10^{-9} \mathrm{ergs} \mathrm{cm}^{-2} \mathrm{~s}^{-1}$. This variation is significant at the $6.4 \sigma$ level. The most recent observation, on 2002 July 29 , found the persistent $2.5-25 \mathrm{keV}$ flux to be higher again, at $(2.19 \pm 0.02) \times 10^{-9} \mathrm{ergs} \mathrm{cm}^{-2} \mathrm{~s}^{-1}$.

\subsection{Variation of $\Delta t$ with $F_{\mathrm{p}}$}

The burst interval measured from the RXTE observations decreased by $40 \%$ between 1997-98 and 2002, while over the same period the mean persistent flux increased by $66 \%$. In order to estimate the bolometric correction to apply to the $2.5-25 \mathrm{keV}$ flux, and thus estimate the ratio $\alpha$ between the integrated persistent flux to the burst fluence (equation 1), we extracted PCA and HEXTE spectra covering the entire observation from five representative pointings (1997 November, 1998 June, 2000 July, 2000 September and 2002 July). We fitted each spectra to a model consisting of a Comptonisation component ("compTT" in XSPEC) and a Gaussian, both attenuated by neutral absorption along the line of sight. The compTT component features a cutoff at around 2.8 times the temperature of the scattering plasma, which we measured as $13.4 \pm 0.5 \mathrm{keV}$ in the mean. In each case we obtained a good fit (mean $\chi^{2} /$ dof $=1.02 \pm 0.13$ ) over the energy range $2.5-60 \mathrm{keV}$. The mean neutral column density was $n_{\mathrm{H}}=(1.52 \pm 0.25) \times 10^{22} \mathrm{~cm}^{-2}$, which is consistent with the mean value derived from the PCA-only pre-burst persistent emission fits. We then measured the (absorbed) flux in the 2.5-25 keV band, and compared this to the flux in the $0.1-200 \mathrm{keV}$ band estimated using an idealized response ${ }^{1}$ in order to extrapolate outside the PCA/HEXTE bands. We found the bolometric correction to be $1.678 \pm 0.016$, where the error represents the standard deviation from the results for the 5 observations.

The resulting variation of the burst recurrence interval, as a function of the bolometric persistent flux, is shown in Fig. 4; qualitatively similar results were found by Cornelisse et al. (2003) from BeppoSAX WFC observations. For the errors on the individual $\Delta t$ measurements from $R X T E$, we adopted a fractional error corresponding to the standard deviation measured by Cocchi et al. (2001) of

1 The response used for extrapolation covered the range $0.1-200 \mathrm{keV}$ with 200 logarithmically spaced energy bins. 


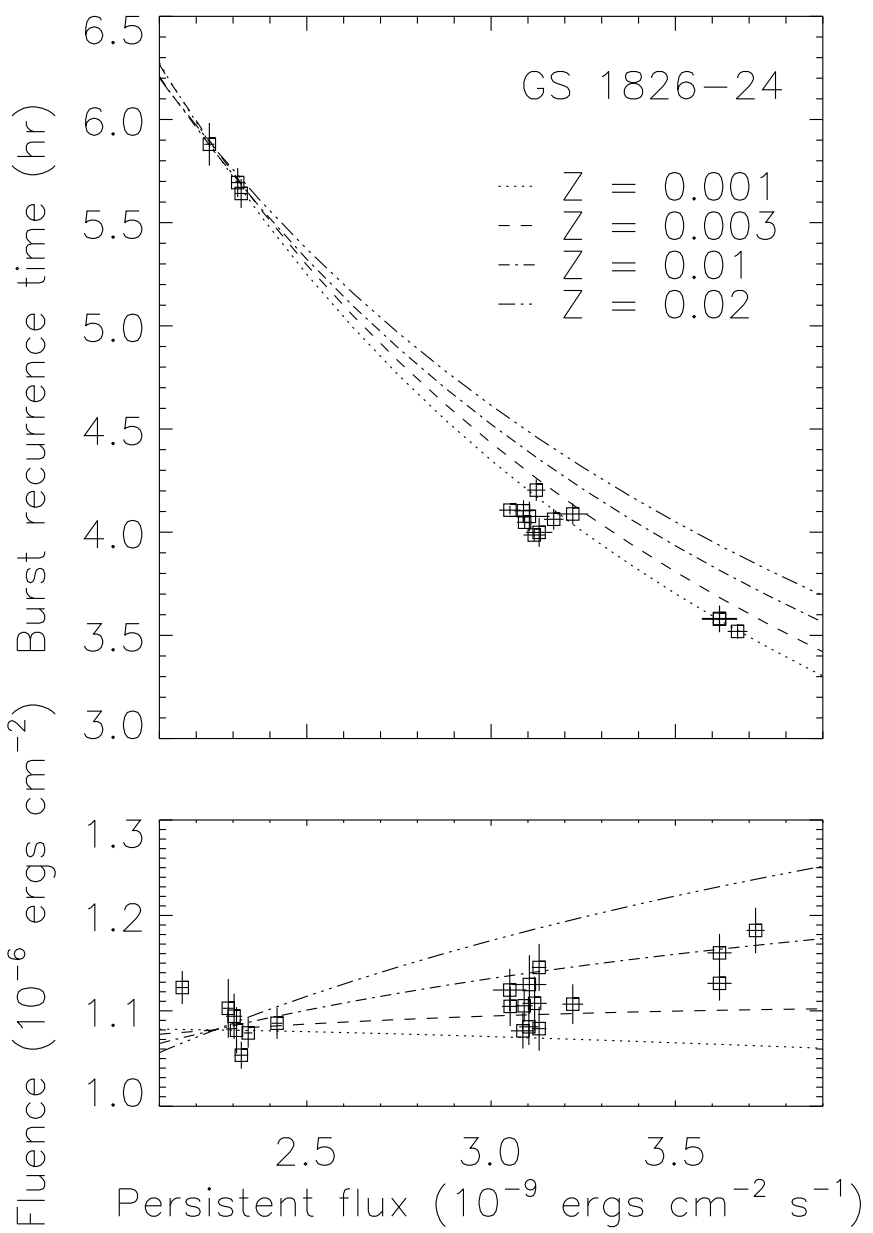

FIG. 4.- Variation of the burst recurrence time (upper panel) and the burst fluence (lower panel) as a function of the estimated bolometric persistent flux in GS 1826-24, from RXTE measurements between 1997-2002. Error bars indicate the $1 \sigma$ errors. The curves show theoretical calculations for a range of metallicities: $Z=0.02,0.01,0.003$, and 0.001 . The solid angle $(R / d)$ and gravitational energy have been chosen in each case to match the observed fluence and recurrence time at $F_{\mathrm{p}}=2.25 \times$ $10^{-9} \mathrm{erg} \mathrm{cm}^{-2} \mathrm{~s}^{-1}$. For $Z=0.02,0.01,0.003$, and 0.001 , this gives $R / d=13,10,8,6 \mathrm{~km} @ 10 \mathrm{kpc}$, and $Q_{\text {grav }}=175,196,211,215 \mathrm{MeV}$ per nucleon.

$0.1 \mathrm{hr}$, for a mean delay time of $5.7 \mathrm{hr}$. This agrees well with the scatter in $\Delta t$ we observe within each epoch. For cases where we miss $n$ or more intervening bursts, we scale the error by $1 / \sqrt{n+1}$, since we assume that we are observing $n+1$ burst intervals in total. From a least-squares fit to $\Delta t$ as a function of $F_{\mathrm{p}}$, using $\Delta t \propto F_{\mathrm{p}}^{\alpha}$ we found a power law index of $\gamma=-1.05 \pm 0.02$.

From the estimated bolometric fluxes we then calculated the ratio $\alpha=E_{\mathrm{p}} / E_{\mathrm{b}}$ from each pair of bursts measured by RXTE (Fig. 5). We found that $\alpha$ varied significantly with epoch, decreasing from $\approx 44$ to $\approx 40$ between 1997-98 and 2002 , as the persistent flux increased. The weighted mean was $41.7 \pm 1.6$.

From the BeppoSAX/WFC observations, Ubertini et al. (1999) derived a value of $60 \pm 7$. Roughly consistent values were determined from BeppoSAX/NFI, RXTE, and $A S C A$ observations, of between 50-54 (in 't Zand et al. 1999; Kong et al. 2000). To understand the discrepancy between these measurements and the value of $\alpha$ calculated in the present study, we note firstly that the previous measurements of the burst fluence from $A S C A$ and $R X T E$ by
Kong et al. (2000) agree well with our measurements. Furthermore, all the persistent flux measurements appear to agree in general once the difference in the energy band is taken into account. However, the burst fluence measured by both the BeppoSAX instruments are around $40 \%$ lower than for $A S C A$ and $R X T E$. The $R X T E / \mathrm{PCA}$ is known to measure fluxes that are systematically $\sim 20 \%$ higher than some other instruments (e.g. Kuulkers et al. 2003); however, this offset is insufficient to explain the discrepancy in the measured fluence. Furthermore, the estimate of $\alpha$ from RXTE should be independent of any systematic flux offset. Despite the substantially higher burst fluence from the RXTE and ASCA measurements of Kong et al. (2000), the calculated $\alpha$ was still close to that measured by BeppoSAX (Ubertini et al. 1999; in 't Zand et al. 1999). This appears to result from the bolometric correction on the $2-10 \mathrm{keV} R X T E / A S C A$ flux, which is not quoted in the Kong et al. (2000) paper but we estimate at 4-6. By comparison, the bolometric correction implied by the 2$10 \mathrm{keV}$ and $0.1-200 \mathrm{keV}$ BeppoSAX/NFI measurements by in 't Zand et al. (1999) is 3.3. From our broadband spectral fits in $\S 3.2$, we estimate a bolometric correction for $R X T E$ flux in the $2-10 \mathrm{keV}$ band as $3.06 \pm 0.02$. Thus, we attribute the higher $\alpha$ measured by Kong et al. (2000) to an excessive bolometric correction factor.

\section{COMPARISON TO THEORETICAL IGNITION MODELS}

In this section, we compare the observed burst properties with theoretical models of type I burst ignition. We calculate ignition conditions following Cumming \& Bildsten (2000), and refer the reader to that paper for details. Since the calculation depends only on the local vertical structure of the layer, we give the results in terms of the local accretion rate per unit area $\dot{m}$, and the mass per unit area or column depth $y$. We assume

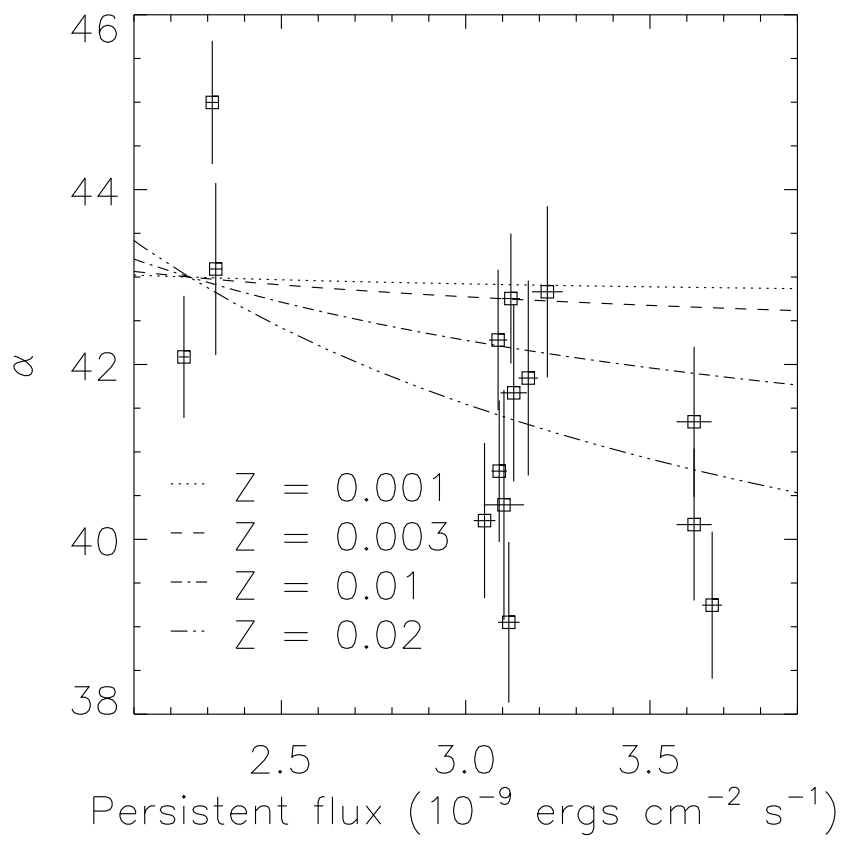

FIG. 5.- Ratio of persistent to burst luminosity $\alpha=L_{\mathrm{p}} / L_{\mathrm{b}}$ (equation 1), calculated from $R X T E$ observations between 1997 and 2002. Error bars represent the estimated $1 \sigma$ uncertainties. The curves show theoretical calculations for the same values of metallicity as in Fig. 4. 
a $1.4 M_{\odot}$ neutron star with radius $R=10 \mathrm{~km}$, giving a surface gravity $g=\left(G M / R^{2}\right)(1+z)=2.45 \times 10^{14} \mathrm{~cm} \mathrm{~s}^{-2}$, where $1+z=\left(1-2 G M / R c^{2}\right)^{-1 / 2}=1.31$ is the gravitational redshift. This value for the redshift is close to that recently measured for EXO 0748-676 $(z \simeq 0.35$; Cottam et al. 2002).

We calculate the temperature profile of the accumulating layer of hydrogen and helium, and adjust its thickness until a thermal runaway occurs at the base. The temperature is mostly set by hydrogen burning via the hot CNO cycle, and therefore the CNO mass fraction $Z$, which we refer to as the metallicity. Our models also include compressional heating and a flux from the crust $F_{\text {crust }}$, but the results are not sensitive to these contributions. A factor of two change in $F_{\text {crust }}$ gives a $2 \%(25 \%)$ change in ignition depth and burst energy for $Z=0.02(Z=0.001)$, with a much smaller change in the trend of these properties with $\dot{m}$. We take $F_{\text {crust }}$ to be constant over the timescale of the observations, i.e. $F_{\text {crust }}=\langle\dot{m}\rangle Q_{\text {crust }}$, where the time-averaged local accretion rate $\langle\dot{m}\rangle$ is set equal to the value for which the burst recurrence time is $5.7 \mathrm{hr}$, and $Q_{\text {crust }}=0.1 \mathrm{MeV}$ per nucleon (Brown 2000).

To calculate the burst energy, we assume complete burning of the $\mathrm{H} / \mathrm{He}$ fuel layer, and that the accreted material covers the whole surface of the star. The total energy is then $4 \pi R^{2} y Q_{\mathrm{nuc}} \xi_{\mathrm{b}}^{-1} /(1+z)$, where $y$ is the ignition column depth, $Q_{\text {nuc }}$ is the energy per gram from nuclear burning, and the factor $\xi_{\mathrm{b}}$ accounts for anisotropic burst emission. We set the latter parameter equal to unity initially, but use it to track where burst anisotropy might play a role. We write $Q_{\text {nuc }}=1.6+4\langle X\rangle \mathrm{MeV}$ per nucleon, where $\langle X\rangle$ is the mass-weighted mean hydrogen fraction at ignition. This expression for $Q_{\text {nuc }}$ assumes $\approx 35 \%$ energy loss due to neutrinos during the rp process (e.g. Fujimoto et al. 1987), and gives $4.4 \mathrm{MeV}$ per nucleon for approximately solar hydrogen abundance $(X=0.7)$.

Table 1 gives ignition conditions for four different metallicities. In each case, we adjust $\dot{m}$ until $\Delta t=(y / \dot{m})(1+$ $z)=5.7 \mathrm{hr}$, as observed in 1997-98. Lower metallicity models have less hot CNO heating during accumulation, giving a larger ignition column and therefore larger $\dot{m}$ to match $\Delta t=5.7 \mathrm{hr}$. We are free to vary $\dot{m}$ because of the uncertainty in the $F_{p}-\dot{m}$ relation. The ratio of observed flux and inferred $\dot{m}$ constrains a combination of the gravitational energy release per nucleon, and the degree of anisotropy in the persistent or burst emission. The persistent flux is

$$
F_{\mathrm{p}}=\frac{L_{\mathrm{X}}}{4 \pi d^{2}}=\frac{\dot{m} Q_{\mathrm{grav}}}{1+z}\left(\frac{R}{d}\right)^{2} \xi_{\mathrm{p}}^{-1},
$$

where $\xi_{\mathrm{p}}$ accounts for anisotropy in the persistent emission, and $Q_{\text {grav }}=c^{2} z /(1+z) \approx G M / R$ is the gravitational energy release per gram. The burst fluence is

$$
E_{b}=\frac{Q_{\mathrm{nuc}} y}{1+z}\left(\frac{R}{d}\right)^{2} \xi_{\mathrm{b}}^{-1} .
$$

Inserting equations (2) and (3) into $\alpha=F_{\mathrm{p}} \Delta t / E_{b}$ gives

$$
\alpha Q_{\mathrm{nuc}}=\left(\frac{\xi_{\mathrm{b}}}{\xi_{\mathrm{p}}}\right) c^{2} z=290 \mathrm{MeV}\left(\frac{z}{0.31}\right)\left(\frac{\xi_{\mathrm{b}}}{\xi_{\mathrm{p}}}\right) .
$$

The last column in Table 1 gives $\alpha Q_{\text {nuc }}$ for the models. Reconciling these values with equation (4) requires $\xi_{\mathrm{b}} / \xi_{\mathrm{p}}=0.5-0.6$, within the range of expected values (e.g. Lapidus \& Sunyaev 1985; Fujimoto 1988), especially since the "anisotropy" factor for the persistent emission includes other effects such as energy radiated outside the X-ray band.

The predicted variations in fluence, recurrence time, and $\alpha$ with $\dot{M}$ are compared to the observations in Figures 4 and 5 . We adjust each curve to match the observed fluence, recurrence time, and $\alpha$ at $F_{\mathrm{p}}=2.25 \times 10^{-9} \mathrm{erg} \mathrm{cm}^{-2} \mathrm{~s}^{-1}$.

The model with $Z=0.02$ (approximately equivalent to solar metallicity) agrees very well with the observed burst energy and variation in $\alpha$ with $\dot{M}$. The predicted burst energy (Table 1) is $5.3 \times 10^{39} \mathrm{ergs}$, corresponding almost exactly to the observed fluence for a distance of $6 \mathrm{kpc}$. The $\approx 10 \%$ variation in $\alpha$ arises because hydrogen burning occurs between bursts as the layer accumulates. In a given fluid element, the time to burn all the hydrogen via the beta-limited hot $\mathrm{CNO}$ cycle is $11 \mathrm{hr}(Z / 0.02)^{-1}\left(X_{0} / 0.7\right)$ (e.g. Bildsten 1998), where $Z$ is the CNO mass fraction, and $X_{0}$ is the accreted hydrogen fraction. This means that as the recurrence time decreases from 5.7 to $4 \mathrm{hr}$, significantly more hydrogen per unit mass is present when the burst ignites, leading to an increase in $Q_{\text {nuc }}$, and corresponding decrease in $\alpha$. Although our models do not address the time evolution of the burst, the variation in mean burst lightcurves shown in Fig. 2 is consistent with a change in the fuel composition.

However, the solar metallicity model is less successful at explaining the observed variations in $\Delta t$ and $E_{\mathrm{b}}$ (Fig. 4). Whereas the observations show a decreasing ignition mass, $\Delta t \propto \dot{M}^{-1.05}$, and $\mathrm{a} \approx 3 \% \mathrm{rms}$ variation in $E_{\mathrm{b}}$, the models have $\Delta t$ decreasing less steeply than $1 / \dot{M}$, and a $\approx 10 \%$ increase in $E_{\mathrm{b}}$ with $\dot{M}$. These variations arise because as recurrence time drops, less hydrogen burning occurs, less helium is present at ignition, and the ignition mass increases.

Extra heating of the accumulating layer would give a reduced ignition mass and better agreement with the observed trends of $\Delta t$ and $E_{\mathrm{b}}$. For the $Z=0.02$ model in Table 1, the extra flux required to match the observed change in $\Delta t$ is $\approx 0.5 \mathrm{MeV}$ per nucleon for an increase of $50 \%$ in $\dot{M}$. This could perhaps be provided by residual heat from the ashes of previous bursts (Taam et al. 1993; Woosley et al. 2003), although time-dependent simulations are required to test this.

A second possibility is that the fraction of the neutron star surface covered by accreted fuel changes with $\dot{M}$, as suggested by Bildsten (2000). We can infer the covering fraction from measurements of the blackbody normalization in the tail of the bursts. We show in Fig. 6 the mean value of $R_{\mathrm{bb}} / d_{10 \mathrm{kpc}}$ between $\approx 20-50 \mathrm{~s}$ after the start of each burst. We staggered the time window over which each average was calculated, to ensure that the mean blackbody temperature over each time interval was approximately constant. We found a significant decrease of $\approx 20 \%$ in the blackbody normalization. A variation of the covering fraction by this amount, and the resulting increase in local accretion rate $\dot{m}$, would go some way to bringing the $\Delta t$ and $E_{\mathrm{b}}$ variations into better agreement. However, the detailed relationship between the measured $R_{\mathrm{bb}}$ and the covering fraction is uncertain. 


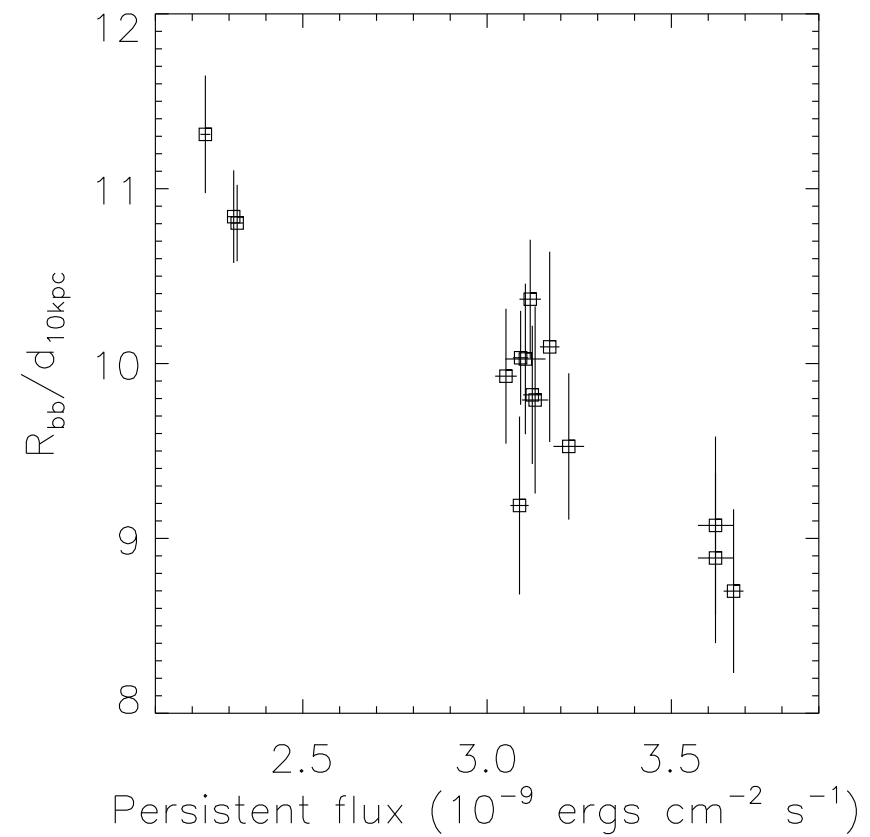

FIG. 6.- Blackbody normalization $R_{\mathrm{bb}} / d_{10 \mathrm{kpc}}$ as a function of bolometric persistent flux, for bursts from GS 1826-24. The normalization was averaged over a time window $\approx 20-50 \mathrm{~s}$ following the start of the burst. Error bars indicate the $1 \sigma$ uncertainties.

While the observed variation in $\alpha$ argues strongly against low metallicity models (which have a constant composition at ignition since little hot CNO burning occurs during accumulation), such models give much better agreement with the observed trends in $\Delta t$ and $E_{\mathrm{b}}$. The constant composition leads to an ignition mass almost independent of $\dot{M}$, giving a constant burst fluence and $\Delta t$ variations close to $1 / \dot{M}$. Low metallicity models overpredict the burst energy, since the reduced CNO heating leads to a larger ignition mass. Accounting for this discrepancy by increasing the distance to the source requires $d \approx 10$ $12 \mathrm{kpc}$, above the current upper limit. However, there are other possible ways to bring the burst energy into agreement. Recent time-dependent simulations of bursts by Woosley et al. (2003) show that leftover hydrogen and CNO nuclei in the ashes of the previous burst lead to extra heating, reducing the critical mass. Also, the fuel may cover only part of the neutron star surface. Table 1 lists the value of $\xi_{\mathrm{b}}^{-1 / 2}(R / d)$ that gives agreement with the observed fluence (eq. [3]), where we now interpret $R$ as a measure of the area covered by accreted material rather than the neutron star radius. For the low metallicity models, we find $\xi_{\mathrm{b}}^{-1 / 2} R \approx 5-6 \mathrm{~km}$ at $6 \mathrm{kpc}$, or covering fractions (assuming a neutron star radius of $10 \mathrm{~km}$ ) of $30-40 \%$.

Finally, we address the sensitivity of our results to the fraction of accreted hydrogen. Recent evolutionary models involving intermediate mass binaries (Podsiadlowski et al. 2002) propose that the secondary has undergone some main sequence hydrogen burning, leading to a relatively hydrogen-poor donor $(X \approx 0.35-0.7)$. As an example, we show results for an accreted hydrogen mass fraction $X_{0}=0.5$ in the lower half of Table 1 . The ignition conditions are not very sensitive to the total amount of hydrogen, since only a small amount of hydrogen burns during accumulation. However, the burst energy is significantly reduced, since $Q_{\text {nuc }}$ depends strongly on the amount of hydrogen versus helium. The variation in burst properties with flux is not a useful way to distinguish the amount of hydrogen, and is almost identical with Figures 4 and 5 for the $X=0.5$ models.

\section{DISCUSSION}

The regularity of the bursting from GS 1826-24 makes it a unique source to compare burst theory and observations. In this paper, we have presented $R X T E / \mathrm{PCA}$ observations of 24 type I X-ray bursts from GS 1826-238. For the first time for this source, we have been able to study the properties of bursts in detail at different mass accretion rates. The bursts from a given epoch have identical lightcurves (Fig. 1 and 2), and show long tails likely powered by rp-process hydrogen burning, as suggested by Bildsten (2000). The bursts from a given epoch are consistent with a single recurrence time, and this varies with accretion rate as $\Delta t \propto \dot{M}^{-1.05}$. The burst fluence increased slightly with the accretion rate, and $\alpha$ decreased by $\approx 10 \%$, with mean value $41.7 \pm 1.6$.

The decrease in $\alpha$ with $\dot{M}$ implies that stable burning of hydrogen occurs between bursts. This requires solar metallicity $(Z \approx 0.02)$ in the accreted layer (Fig. 5). Solar metallicity models also give good agreement with the observed burst energies, as pointed out by Bildsten (2000). However, the small variation in fluence, and $\Delta t$ variation with $\dot{M}$ is difficult to explain with solar metallicity models, which predict $10 \%$ variations in fluence, and $\Delta t$ decreasing less steeply than $1 / \dot{M}$. Low metallicity models $(Z \sim 0.001)$ naturally explain the small variation in burst fluence, and $\approx 1 / \dot{M}$ scaling of $\Delta t$, since little CNO burning occurs between bursts giving an ignition mass almost independent of $\dot{M}$. However, the observed $\alpha$ variations with $\dot{M}$ rule out low metallicity models.

There are several possible ways to reconcile the solar metallicity models with the observations of $\Delta t$ and $E_{\mathrm{b}}$. Studies of timing and spectra of LMXBs indicate that $L_{X}$ is not always a good indicator of $\dot{M}$ (e.g. van der Klis et al. 1990), we have assumed $L_{X} \propto \dot{M}$ here. Extra heating of the accumulating layer would act to reduce the critical mass and bring the observations and theory into agreement. One possibility is that residual heat from the ashes of previous bursts heats the layer (Taam et al. 1993; Woosley et al. 2003), time-dependent simulations are needed to test this. If the fraction of the neutron star surface covered by fuel changes with $\dot{M}$, the changing local accretion rate per unit area could also reconcile the models and observations. We find that the blackbody radius $R_{\mathrm{bb}}$ in the tail of the bursts decreased by $\approx 20 \%$ between the observed epochs. If this indicates a change in covering fraction, it would almost be enough to explain the discrepancy. However, the covering fraction decrease with $\dot{M}$ is opposite to the increase suggested by Bildsten (2000) to explain trends in burst properties. Furthermore, $R_{\mathrm{bb}}$ is at best an uncertain measure of the emitting area. Due to variations in the atmospheric opacity with photon energy, the measured blackbody temperature is generally higher than the effective temperature (e.g. London et al. 1986; Shaposhnikov et al. 2003). Consequently, $R_{\mathrm{bb}}$ is expected to give an underestimate of the emitting area, by a factor which depends on the detailed structure and composition 
of the NS atmosphere. Better $R / d$ measurements could be obtained with high resolution spectra and appropriate model atmospheres.

Woosley et al. (2003) have recently calculated timedependent multi-zone burst models including a large nuclear reaction network. They show that both the rise and decay times of the burst depend sensitively on nuclear properties in the rp-process path, and in addition find burst rise times of several seconds, in good agreement with the observations. There is much to learn from a detailed comparison of time-dependent simulations with the burst lightcurves. In particular, the variation in decay time with accretion rate may directly reflect the changing abundance of hydrogen at ignition.

Many bursters show a transition from regular, frequent bursts with long durations at low $\dot{m}$, to irregular, infrequent and short bursts at high $\dot{m}$, a behavior first discovered by EXOSAT (van Paradijs et al. 1988). By comparing BeppoSAX/WFC observations of nine regular type I bursters, Cornelisse et al. (2003) propose that this behavior is common to all bursters, and that the transition occurs at a universal luminosity $L_{X} \approx 2 \times 10^{37} \mathrm{erg} \mathrm{s}^{-1}$. While the physics of the transition to irregular bursting is not understood (e.g. see discussion by Bildsten 2000), we have shown in this paper that the regular bursting seen in GS 1826-24 is well-understood as being due to helium ignition in a hydrogen-rich environment. This argues that other bursters such as KS 1731-260 are in this burning regime when regular bursting is seen (and not bursting via unstable hydrogen ignition, as suggested by van Paradijs et al. 1988 and Cornelisse et al. 2003). BeppoSAX found a peak burst rate for KS 1731-260 (the burster with the largest range in observed $\dot{m}$ 's) of $\approx 9$ bursts per day. Extrapolating the observed $1 / \dot{M}$ dependence of $\Delta t$, we expect GS 1826-24 to reach this burst rate, and perhaps transition to irregular bursting at $F_{p} \approx 4.6 \times 10^{-9} \mathrm{erg} \mathrm{cm}^{-2} \mathrm{~s}^{-1}$. Further monitoring of the burst behavior of GS 1826-24 will hopefully probe the physics of the transition to irregular bursting.

This research has made use of data obtained through the High Energy Astrophysics Science Archive Research Center Online Service, provided by the NASA/Goddard Space Flight Center. This work was supported in part by NASA under grants NAG 5-9184 and NAG 5-8658, contract NAS5-30720 and by the National Science Foundation under grant PHY99-07949. L. B. is a Cottrell Scholar of the Research Corporation. A.C. is supported by NASA Hubble Fellowship grant HF-01138 awarded by the Space Telescope Science Institute, which is operated for NASA by the Association of Universities for Research in Astronomy, Inc. under contract NAS 5-26555.

\section{REFERENCES}

Barret, D., Motch, C., \& Pietsch, W. 1995, A\&A, 303, 526

Becker, R. H., Pravdo, S. H., Serlemitsos, P. J., Swank, J. H., \& Hoffman, J. 1976, IAU Circ., 2953

Bildsten, L. 1998, in The Many Faces of Neutron Stars, ed. R. Buccheri, J. van Paradijs, \& A. Alpar (Dordrecht: Kluwer), 419

Bildsten, L. 2000, in Cosmic Explosions, the 10th Annual October Astrophysics Conference, Maryland, October 11-13 1999, AIP Conf. 522, ed. S. Holt \& W. Zhang (Woodbury NY: AIP)

Brown, E. F. 2000, ApJ, 531, 988

Cocchi, M., Bazzano, A., Natalucci, L., Ubertini, P., Heise, J., Kuulkers, E., \& in't Zand, J. J. M. 2001, Advances in Space Research, 28, 375

Cornelisse, R., in't Zand, J. J. M., Verbunt, F., Kuulkers, E., Heise, J., den Hartog, P. R., Cocchi, M., Natalucci, L., Bazzano, A., \& Ubertini, P. 2003, A\&A, 405, 1033

Cottam, J., Paerels, F., \& Mendez, M. 2002, Nature, 420, 51

Cumming, A. 2003, ApJ, in press (astro-ph/0306245)

Cumming, A. \& Bildsten, L. 2000, ApJ, 544, 453

Fujimoto, M. Y. 1988, ApJ, 324, 995

Fujimoto, M. Y., Sztajno, M., Lewin, W. H. G., \& van Paradijs, J. 1987, ApJ, 319, 902

Gottwald, M., Haberl, F., Parmar, A. N., \& White, N. E. 1986, ApJ, 308,213

Hanawa, T., Sugimoto, D., \& Hashimoto, M.-A. 1983, PASJ, 35, 491

Homer, L., Charles, P. A., \& O'Donoghue, D. 1998, MNRAS, 298, 497

in 't Zand, J. J. M., Heise, J., Kuulkers, E., Bazzano, A., Cocchi, M., \& Ubertini, P. 1999, A\&A, 347, 891

Jahoda, K., Swank, J. H., Giles, A. B., Stark, M. J., Strohmayer, T., Zhang, W., \& Morgan, E. H. 1996, Proc. SPIE, 2808, 59

Koike, O., Hashimoto, M., Arai, K., \& Wanajo, S. 1999, A\&A, 342, 464

Kong, A. K. H., Homer, L., Kuulkers, E., Charles, P. A., \& Smale, A. P. 2000, MNRAS, 311, 405

Kuulkers, E., den Hartog, P. R., in't Zand, J. J. M., Verbunt, F. W. M., Harris, W. E., \& Cocchi, M. 2003, A\&A, 399, 663

Kuulkers, E., Homan, J., van der Klis, M., Lewin, W. H. G., \& Méndez, M. 2002, A\&A, 382, 947

Langmeier, A., Sztajno, M., Hasinger, G., Truemper, J., \& Gottwald, M. 1987, ApJ, 323, 288

Lapidus, I. I. \& Sunyaev, R. A. 1985, MNRAS, 217, 291

Levine, A. M., Bradt, H., Cui, W., Jernigan, J. G., Morgan, E. H., Remillard, R., Shirey, R. E., \& Smith, D. A. 1996, ApJ, 469, L33
London, R. A., Taam, R. E., \& Howard, W. M. 1986, ApJ, 306, 170

Podsiadlowski, P., Rappaport, S., \& Pfahl, E. D. 2002, ApJ, 565, 1107

Rembges, F., Freiburghaus, C., Rauscher, T., Thielemann, F., Schatz, H., \& Wiescher, M. 1997, ApJ, 484, 412

Rothschild, R. E., Blanco, P. R., Gruber, D. E., Heindl, W. A., MacDonald, D. R., Marsden, D. C., Pelling, M. R., Wayne, L. R., \& Hink, P. L. 1998, ApJ, 496, 538

Schatz, H., Aprahamian, A., Barnard, V., Bildsten, L., Cumming, A., Ouellette, M., Rauscher, T., Thielemann, F.-K., \& Wiescher, M. 2001, Physical Review Letters, 86, 3471

Schatz, H., Aprahamian, A., Goerres, J., Wiescher, M., Rauscher, T., Rembges, J. F., Thielemann, F.-K., Pfeiffer, B., Moeller, P., Kratz, K.-L., Herndl, H., Brown, B. A., \& Rebel, H. 1998, Phys. Rep., 294,167

Shaposhnikov, N., Titarchuk, L., \& Haberl, F. 2003, ApJ, 593, L35

Strohmayer, T. \& Bildsten, L. 2003, in Compact Stellar X-Ray Sources, ed. W. H. G. Lewin \& M. van der Klis (Cambridge University Press), in press; astro-ph/0301544

Taam, R. E., Woosley, S. E., Weaver, T. A., \& Lamb, D. Q. 1993, ApJ, 413, 324

Tanaka, Y. 1989, in Proc. 23rd ESLAB Symposium on Two Topics in X-ray Astronomy, Bologna, Italy, 13-20 September, ed. J. Hunt \& B. Battrick No. SP-296 (ESTEC, Noordwijk, The Netherlands: ESA), 3-13

Ubertini, P., Bazzano, A., Cocchi, M., Natalucci, L., Heise, J., Jager, R., in 't Zand, J., Muller, J. M., Smith, M., Celidonio, G., Coletta, A., Ricci, R., Giommi, P., Ricci, D., Capalbi, M., Menna, M. T., \& Rebecchi, S. 1997, IAU Circ., 6611

Ubertini, P., Bazzano, A., Cocchi, M., Natalucci, L., Heise, J., Muller, J. M., \& in 't Zand, J. J. M. 1999, ApJ, 514, L27

van der Klis, M., Hasinger, G., Damen, E., Penninx, W., van Paradijs, J., \& Lewin, W. H. G. 1990, ApJ, 360, L19

van Paradijs, J., Penninx, W., \& Lewin, W. H. G. 1988, MNRAS, 233,437

Verbunt, F. \& van den Heuvel, E. P. J. 1995, in X-ray Binaries, ed. W. H. G. Lewin, J. van Paradijs, \& E. P. J. van den Heuvel (Cambridge: Cambridge University Press), 457-494

Wallace, R. K. \& Woosley, S. E. 1981, ApJS, 45, 389

Woosley, S. E., Heger, A., Cumming, A., Hoffman, R. D., Pruet, J., Rauscher, T., Schatz, H., Brown, B. A., \& Wiescher, M. 2003, ApJ, submitted (astro-ph/0307425) 
TABLE 1

IGNITION CONDITIONS FOR BURSTS WITH $\Delta t=5.7$ hours $^{\mathrm{a}}$

\begin{tabular}{|c|c|c|c|c|c|c|c|c|c|}
\hline$Z$ & $\dot{m}_{4}$ & $y_{8}$ & $T_{8}$ & $X$ & $\langle X\rangle$ & $Q_{\text {nuc }}{ }^{\mathrm{b}}$ & $\begin{array}{c}E_{\mathrm{burst}}{ }^{\mathrm{c}} \\
\left(10^{39} \text { erg }\right)\end{array}$ & $\begin{array}{c}R / d^{\mathrm{d}} \\
(\mathrm{km} @ 6 \mathrm{kpc})\end{array}$ & $\alpha Q_{\text {nuc }}$ \\
\hline \multicolumn{10}{|c|}{ Accreted $\mathrm{H}$ fraction $X_{0}=0.7$} \\
\hline 0.02 & 0.97 & 1.5 & 2.2 & 0.40 & 0.55 & 3.8 & 5.3 & 9.6 & 152 \\
\hline 0.01 & 1.4 & 2.1 & 2.1 & 0.55 & 0.62 & 4.1 & 8.0 & 7.8 & 164 \\
\hline 0.003 & 2.1 & 3.3 & 2.0 & 0.65 & 0.68 & 4.3 & 13 & 6.1 & 172 \\
\hline 0.001 & 2.8 & 4.3 & 1.9 & 0.68 & 0.69 & 4.4 & 17 & 5.3 & 175 \\
\hline \multicolumn{10}{|c|}{ Accreted $\mathrm{H}$ fraction $X_{0}=0.5$} \\
\hline 0.02 & 0.92 & 1.4 & 2.1 & 0.20 & 0.35 & 3.0 & 4.0 & 11 & 120 \\
\hline 0.01 & 1.2 & 1.9 & 2.0 & 0.35 & 0.42 & 3.3 & 5.8 & 9.2 & 132 \\
\hline 0.003 & 1.8 & 2.9 & 1.9 & 0.45 & 0.48 & 3.5 & 9.3 & 7.3 & 140 \\
\hline 0.001 & 2.4 & 3.8 & 1.8 & 0.48 & 0.49 & 3.6 & 12 & 6.3 & 143 \\
\hline
\end{tabular}

Note. - The first six columns are: CNO mass fraction $Z$; local rest mass accretion rate at the surface of the star $\dot{m}_{4}=\dot{m} / 10^{4} \mathrm{~g} \mathrm{~cm}^{-2} \mathrm{~s}^{-1}$; ignition column depth $y_{8}=y / 10^{8} \mathrm{~g} \mathrm{~cm}^{-2}$; ignition temperature $T_{8}=T / 10^{8} \mathrm{~K}$; hydrogen mass fraction at the base $X$; mean hydrogen mass fraction $\langle X\rangle=\int d y X(y) / y$.

${ }^{\mathrm{a}} \Delta t=(y / \dot{m})(1+z)$ is the recurrence time measured by an observer at infinity.

${ }^{\mathrm{b}}$ Nuclear energy release, $Q_{\text {nuc }}=1.6+4.0\langle X\rangle \mathrm{MeV}$ per nucleon.

${ }^{\mathrm{c}}$ Predicted burst energy assuming the fuel covers the surface of the star, $E_{\text {burst }}=$ $4 \pi R^{2} y Q_{\text {nuc }} \xi_{\mathrm{b}}^{-1} /(1+z)$ (we take $R=10 \mathrm{~km}, z=0.31, \xi_{\mathrm{b}}=1$ ). The observed burst energy is $4.9 \times 10^{39} \mathrm{erg}(d / 6 \mathrm{kpc})^{2}$.

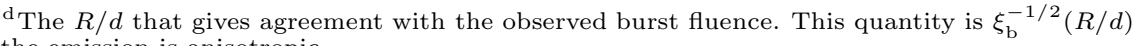
if the emission is anisotropic.

${ }^{\text {e}}$ We adopt the observed value $\alpha=40$ 